\title{
Produção científica da enfermagem dos hospitais de ensino acerca da cultura de segurança do paciente
}

\section{Scientific production of the nursing of teaching hospitals about patient safety culture}

\author{
Tavane Menezes Costa $^{1} \bullet$ Zenith Rosa Silvino $^{2}$
}

\begin{abstract}
RESUMO
A Cultura de Segurança da Instituição deve preconizar a substituição da culpa e da punição pela oportunidade de aprender com as falhas e melhorar a atenção à saúde. Objetivo: Conhecer a produção científica da enfermagem dos hospitais de ensino sobre Cultura de Segurança do paciente. Metodologia: Revisão Integrativa tendo como questão de pesquisa do estudo Qual a produção científica da enfermagem dos hospitais de ensino referente à Cultura de Segurança do paciente? A coleta de dados ocorreu de jan a mar/16, em bases de dados indexadas e online no período entre 2004 e 2016. Foram encontrados 769 artigos, e após aplicação dos critérios de inclusão e exclusão, foram selecionados 13. Resultados: Os artigos foram classificados em duas categorias: Avaliação da Cultura de Segurança do paciente por meio da realização de pesquisas sobre a temática e A pesquisa científica e sua contribuição na avaliação da Cultura de Segurança do paciente. Conclusão: Observou-se um aumento progressivo nas pesquisas de enfermagem nessa temática utilizando a produção científica como importante estratégia para mudanças de paradigmas institucionais e dentro da própria equipe de enfermagem. As lacunas apontadas nesse estudo poderão alavancar mais pesquisas nessa temática.
\end{abstract}

Palavras-chave: Enfermagem; Segurança do paciente; Hospital.

\section{ABSTRACT}

The safety culture of the institution must recommend the replacement of the guilt and punishment for the opportunity to learn from the failures and improve health care. Objective: to know the scientific production of the nursing of teaching hospitals on patient safety Culture. Methodology: Integrative Review with the question of research study Which the scientific production of the nursing of teaching hospitals concerning patient safety Culture? Data collection took place from jan to mar/16, indexed databases and on line at the time period between the 2004 2016.0. Found 769 articles and after application of the criteria of inclusion and exclusion, 13 selected articles. Results: The articles have been classified into 2 categories: Evaluation of patient safety Culture by conducting research on the thematic and Scientific research and its contribution in assessing the culture of patient safety. Conclusion: There is progressive increase in nursing research in this theme using the scientific literature as an important strategy for institutional paradigms and changes within the nursing staff. The gaps identified in this study can leverage more research in this theme.

Keywords: Nursing; Patient safety; Hospital.

Enfermeira pediatra, Mestranda do Programa Acadêmico em Ciências do Cuidado em Saúde na linha de pesquisa O cuidado no seu contexto sociocultural da Escola de Enfermagem Aurora de Afonso Costa da Universidade Federal Fluminense. Coordenadora da Educação Permanente de Enfermagem do Instituto de Puericultura e Pediatria Martagão Gesteira da Universidade Federal do Rio de Janeiro e Enfermeira do Programa de Hipertensão e Diabetes da Secretaria Municipal de Saúde do Rio de Janeiro. E-mail: menezestc@globo.com.

${ }^{2}$ Enfermeira, Orientadora, Prof $^{\mathrm{a}} \mathrm{Dr}^{\mathrm{a}}$ Titular do Departamento de Administração em Enfermagem da Escola de Enfermagem Aurora de Afonso Costa da Universidade Federal Fluminense, atuando na graduação, especialização, Mestrado Profissional e Acadêmico e no Doutorado. Graduação em Direito (1988). Avaliadora Institucional e de Cursos do INEP. Membro Titular da Academia Brasileira de Administração Hospitalar. Integra o Corpo Editorial da Revista Science Rise, Revista de Enfermagem UFPE On Line e Revista Brasileira de Docência, Ensino e Pesquisa em Enfermagem, é consultora ad hoc de renomadas revistas. E-mail:zenithrosa52@gmail.com.

Artigo baseado na Dissertação de Mestrado intitulada "Cultura de Segurança do paciente pediátrico na perspectiva da equipe de enfermagem" de autoria da Mestranda Tavane Menezes Costa do Programa acadêmico em Ciência do Cuidado em saúde da Escola de Enfermagem Aurora de Afonso Costa da Universidade Federal Fluminense, em Niterói, Rio de Janeiro, RJ, 2016. 


\section{INTRODUÇÃO}

A preocupação constante com a qualidade do cuidado e com a segurança do paciente nas instituições de saúde se propagou mundialmente. 0 movimento voltado para a segurança do paciente teve seu início na última década do século XX, após a publicação do relatório do Institute of Medicine (IOM) dos EUA que publicou resultados de vários estudos os quais evidenciaram a grave situação da assistência à saúde daquele país. Dados revelaram que de 33,6 milhões de internações 44.000 a 98.000 pacientes, aproximadamente, morreram em consequência de eventos adversos.

A partir desse alerta do IOM, a Organização Mundial de Saúde (OMS), preocupando-se com a segurança do paciente, elegeu esse tema como de alta prioridade entre os países membros no ano de 2000. Em 2004, lançou a Aliança Mundial para a Segurança do Paciente com foco central na criação dos Desafios Globais para a Segurança do paciente e para liderar internacionalmente os programas de segurança do paciente ${ }^{1}$, além de fomentar pesquisas nessa temática nos países em desenvolvimento, entre outros.

Em 2009, a OMS cria então a Agência Nacional para a Segurança do Paciente e desenvolve a Classificação Internacional de Segurança do Paciente - Taxinomia, que define, entre outros, Eventos Adversos (EAs) os incidentes resultantes de um dano desnecessário à saúde ${ }^{1}$. As pesquisas apontam que os EAs ocorrem pelas fragilidades nos processos, no sistema organizacional, na ausência de liderança e nos fatores relacionados ao comportamento humano. 0 erro é não intencional, multifatorial e produto de sistemas mal estruturados e mal desenhados².

No Brasil, um estudo de 2009 revelou que a incidência de eventos adversos foi de $7,6 \%$ (84 de 1.103$)^{4}$, e a proporção de eventos adversos evitáveis foi de 66,7\% (56 de 84 pacientes). Tal estudo evidencia que os EAs nos três hospitais de ensino no Rio de Janeiro ocorreram de forma semelhante a dos estudos internacionais. No entanto, a proporção de eventos adversos evitáveis foi muito maior nos hospitais brasileiros. 0 mesmo estudo mostrou que tais EAs têm expressivo impacto desnecessário nos gastos hospitalares e razões para supor que os dados estejam subestimados, ou seja, podem ser maiores ainda ${ }^{5}$.

Sabemos que erros acontecem, por isso precisamos tornar o ambiente de prestação de assistência mais seguro ao assumir que é de alto risco. A Cultura de Segurança tem a prevenção de erros como prioridade para todos no processo de trabalho e pode ser definida como:

Conjunto de valores, atitudes, competências e comportamentos que determinam o comprometimento com a gestão da saúde e da segurança, substituindo a culpa e a punição pela oportunidade de aprender com as falhas e melhorar a atenção à saúde 3 .

Ou seja, aprender com os erros, não punindo culpados e sim remodelando o sistema para reduzi-los levará a uma Cultura de Segurança que permitirá continuamente o gerenciamento de riscos 6 .

Nesse contexto, a enfermagem, além de ser uma profissão voltada para o cuidar, é uma das maiores forças de trabalho em saúde no Brasil e no mundo, portanto precisa se empoderar dessa posição vantajosa e modificar a realidade da segurança do paciente ${ }^{7}$ Sabe-se que o fazer da Enfermagem não é apenas técnico, não engloba somente rotinas executadas em série, utiliza-se também de aspectos comportamentais (de adaptações e independências, por exemplo) e das relações humanas (interações enfermeiro/ paciente, orientações, diálogos, famílias etc) ${ }^{8}$. Soma-se a posição vantajosa da enfermagem em relação à segurança do paciente, uma vez que se encontra em um leito?.

Considerando que os Hospitais de ensino têm por missão produzir, sistematizar e socializar o conhecimento e o saber gerados na área da sáude ${ }^{10}$, a enfermagem atuante nesses hospitais pode contribuir de forma ímpar na prestação do cuidado seguro e livre de dano aos pacientes, utilizando a pesquisa cientifica como principal estratégia para identificação, interpretação, implementação e avaliação do cuidado prestado.

Diante do exposto, o objetivo desse estudo é conhecer a produção científica de enfermagem dos hospitais de ensino acerca da cultura de segurança do paciente, utilizando como método de pesquisa a Revisão Integrativa.

\section{MÉTODO}

Para atender o objetivo proposto, esta pesquisa usou como método a Revisão Integrativa, delimitando-se as seguintes etapas percorridas: identificação do problema ou questão de pesquisa; estabelecimento de critérios para inclusão e exclusão de estudos/amostragem ou busca na literatura; definição das informações a serem extraídas dos estudos selecionados; avaliação dos estudos incluídos na revisão integrativa; interpretação dos resultados; apresentação da revisão/síntese do conhecimento ${ }^{11}$ -

A Revisão Integrativa é um método de análise de pesquisas utilizado para promover a síntese do conhecimento em um determinado assunto e incluir estudos com diferentes abordagens metodológicas, além de mostrar lacunas do conhecimento que necessitam ser preenchidas com a realização de novos estudos. Por meio desse método é possível melhorar a prática dos enfermeiros, pois o conhecimento é construído com base em evidências, assim, melhora-se a qualidade da assistência da enfermagem e se facilitam as tomadas de decisões ${ }^{12}$.

Dessa forma, a questão de pesquisa desse estudo é a seguinte: Qual a produção científica da enfermagem dos hospitais de ensino referente à Cultura de Segurança do paciente?

A coleta de dados ocorreu entre janeiro a março de 2016. Foram acessados artigos publicados no período de 2004 (ano da criação da Aliança Mundial para a Segurança do Paciente) a 2016, indexados na base de dados por meio 
da Biblioteca Virtual em Saúde (BVS), a qual abrange: LILACS, BDENF, IBECS, MEDLINE e CUMED. A SciELO também foi escolhida por se tratar de uma biblioteca eletrônica em ciências da saúde, que disponibiliza vasta coleção de periódicos brasileiros com textos completos e on-line. Na sequência, foi utilizada a terminologia em saúde consultada nos Descritores em Ciências da Saúde (DeCS): Enfermagem, Hospital, Segurança do paciente com o operador booleano "AND".

Os critérios de inclusão foram: artigos publicados em língua portuguesa, inglesa ou espanhola, em bases de dados nacionais e internacionais que abordassem a temática sobre Cultura de Segurança do Paciente ou Clima de segurança, manuscritos que tivessem Enfermeiros entre os autores e hospitais universitários como cenários, disponíveis na íntegra, no período compreendido entre 2004 e 2016. Os artigos encontrados de forma duplicada foram considerados apenas uma vez.

Como critérios de exclusão, artigos da categoria cartas ao editor, relatos de experiência, dissertações, teses, revisões integrativas e sistemáticas, bem como estudos que não abordassem a temática relevante ao objetivo desse estudo.

A análise e síntese dos dados retirados dos artigos foram realizadas de forma descritiva, possibilitando observar, contar, descrever e catalogar os dados, com o intuito de unir o conhecimento produzido sobre o tema explorado na revisão ${ }^{13}$.

A seguir observa-se o fluxograma explicativo da seleção amostral.

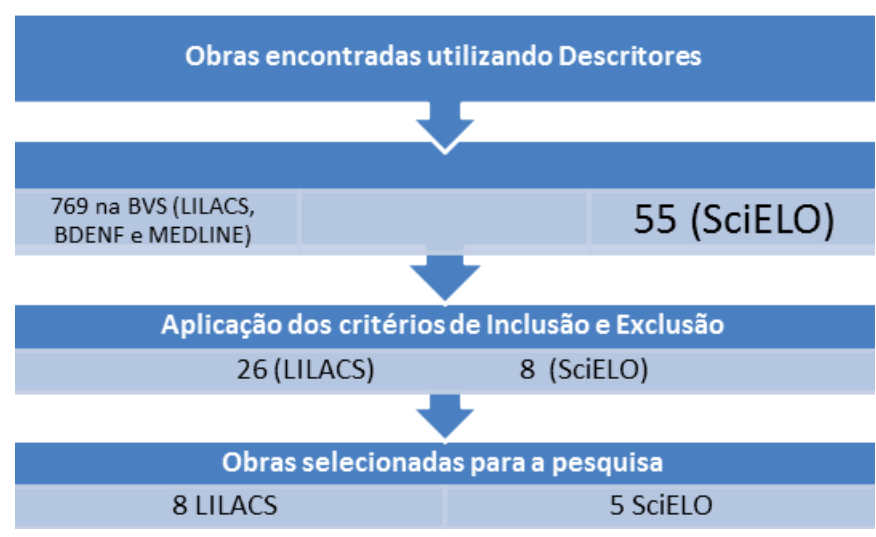

Figura 1: Fluxograma das etapas para seleção dos Artigos. Rio de Janeiro, RJ, Brasil, 2016.

\section{RESULTADOS E DISCUSSÃO}

Em conformidade com os critérios de inclusão e exclusão, a amostra final elencou treze artigos para análise.

De acordo com análise das variáveis mencionadas e em conformidade com o objetivo desse estudo, observou-se que cinco artigos foram encontrados na SciElo biblioteca eletrônica e oito na base de dados eletrônica Lilacs, todos publicados em português e inglês. Os manuscritos foram publicados nos seguintes periódicos conforme tabela 1 abaixo.

Tabela 1. Distribuição dos artigos por Periódicos e ano de publicação. Rio de Janeiro, RJ, 2016.

\begin{tabular}{l|c|c}
\hline \multicolumn{1}{c|}{ Periódico } & $\begin{array}{c}\text { Ano de } \\
\text { publicação }\end{array}$ & Quantidade \\
\hline \multirow{2}{*}{ Revista Acta Paulista } & 2010 & 1 \\
\cline { 2 - 3 } & 2012 & 1 \\
\hline Revista Ciencia y Enfermeria & 2013 & 1 \\
\hline \multirow{2}{*}{ Revista Gaúcha de Enfermagem } & 2013 & 1 \\
\cline { 2 - 3 } & 2014 & 1 \\
\cline { 2 - 3 } & 2015 & 1 \\
\hline Revista Mineira de Enfermagem & 2015 & 1 \\
\hline Revista da Esc. Enf. Anna Nery & 2015 & 1 \\
\hline Revista da Esc. Enf. da USP & 2015 & 1 \\
\hline Revista Brasileira de Enfermagem & 2015 & 1 \\
\hline \multirow{2}{*}{ Revista Latino Americana } & 2015 & 1 \\
\cline { 2 - 3 } & & 1 \\
\hline Revista Texto e Contexto & 2015 & 1 \\
\hline
\end{tabular}

Quanto aos objetivos propostos pelas publicações, permearam o interesse em Conhecer e Avaliar a percepção dos trabalhadores de enfermagem acerca do Clima de Segurança do paciente e Cultura de segurança; Investigar e analisar aspectos relevantes em relação à Identificação do Paciente, à Lavagem das Mãos e ao Checklist de Cirurgias Seguras e, por último, Identificar e Analisar condutas adotadas pelos profissionais frente à ocorrência de erros de medicação.

Em relação ao ano de publicação, constatou-se que a maioria dos artigos foi publicada nos últimos cinco anos, com aumento significativo de publicações em 2015, com 8 (oito) dos 13 (treze) artigos elencados.

Os treze artigos selecionados foram lidos detalhadamente e analisados, conforme apresentados nas tabelas 1 e 2 .

Para interpretação, análise e discussão, os resultados foram divididos em dois eixos temáticos: 1- Avaliação da Cultura de Segurança do paciente por meio da contribuição da pesquisa sobre a temática; 2 - A pesquisa científica e sua contribuição na avaliação da Cultura de Segurança do paciente.

\section{1 - Avaliação da Cultura de Segurança do paciente por meio da realização de pesquisas sobre a temática}

Nessa categoria destacam-se os artigos que discutem as várias ferramentas usadas para Conhecer e Avaliar a Cultura de Segurança do paciente nos Hospitais de Ensino. O Quadro 1 apresenta os sete artigos que a compõem, dispostos pelas Variáveis: Título, Ano, Autores, Idioma, Objetivos e Desfecho. 
A discussão referente à Avaliação da Cultura de Segurança do Paciente em diferentes Hospitais de Ensino em vários estados do país demonstra o interesse progressivo com pesquisas nessa temática por parte dos profissionais de saúde, em especial pelos Enfermeiros.

No artigo $1^{14}$, os autores utilizaram os dois questionários mais empregados em todo mundo para mensuração da Cultura de segurança, que são o "Hospital Survey on Patient Safety Culture (HSOPSC) ${ }^{15}$ e (SAO) Safety Attitudes Questionnaire ${ }^{16}$. Eles avaliaram a percepção dos profissionais de saúde das UTIs (Unidades de Tratamentos Intensivos) Adulto, pediátrica e neonatal de um Hospital de ensino no interior de São Paulo e a relação entre os dois Instrumentos. Entre as UTIs, a UTI Adulto apresentou menores pontuações para a maioria dos domínios do SAQ e do HSOPSC. Foi demonstrado pelo estudo diferenças de percepções quanto à segurança do paciente entre as UTIs, sugerindo a existência de micro-culturas locais, além de o estudo não demonstrar que o SAQ e o HSOPSC sejam equivalentes.

Quadro 1. Avaliação da Cultura de Segurança do paciente através da realização de pesquisas sobre a temática. Rio de Janeiro, RJ, Brasil, 2016

\begin{tabular}{|c|c|c|c|c|}
\hline $\begin{array}{l}\text { Identificação } \\
\text { do Artigo }\end{array}$ & $\begin{array}{c}\text { Título do artigo/ } \\
\text { Idioma/Ano }\end{array}$ & Autores & Objetivos & Desfecho \\
\hline 1 & $\begin{array}{l}\text { Cultura e Clima } \\
\text { organizacional para } \\
\text { segurança do paciente } \\
\text { em Unidades de terapia } \\
\text { intensiva }{ }^{14} \\
\text { Português e inglês } \\
2015\end{array}$ & $\begin{array}{l}\text { Santiago THR } \\
\text { Tussini RNT/ } \\
\end{array}$ & $\begin{array}{l}\text { Avaliar a percepção dos } \\
\text { profissionais de saúde sobre o } \\
\text { clima e cultura de segurança do } \\
\text { paciente em unidades de terapia } \\
\text { intensiva (UTI) e a relação } \\
\text { entre os instrumentos Hospital } \\
\text { Survey on Patient Safety Culture } \\
(\text { HSOPSC) })^{15} \text { e Safety Attitudes } \\
\text { Questionnaire (SAQ) }\end{array}$ & $\begin{array}{l}\text { As escalas apresentaram boa } \\
\text { confiabilidade. Maiores fragilidades } \\
\text { para a segurança do paciente foram } \\
\text { observadas nos domínios "condições } \\
\text { de trabalho" e "percepções da } \\
\text { gerência" do SAQ e "resposta não } \\
\text { punitiva aos erros" do HSOPSC. As } \\
\text { fortalezas no SAQ foram o "clima de } \\
\text { trabalho em equipe" e a "satisfação } \\
\text { no trabalho" e para o HSOPSC } \\
\text { "expectativas e ações de promoção } \\
\text { de segurança supervisores/gerentes" } \\
\text { e "aprendizado organizacional e } \\
\text { melhoria mútua". Na UTI Neonatal } \\
\text { houve maior satisfação no trabalho } \\
\text { do que nas demais UTI. A UTI Adulto } \\
\text { apresentou menores pontuações para } \\
\text { a maioria dos domínios do SAQ e } \\
\text { HSOPSC. A correlação entre as escalas } \\
\text { foi de força moderada (r=0,66). }\end{array}$ \\
\hline 2 & $\begin{array}{l}\text { Cultura de segurança } \\
\text { do paciente em } \\
\text { unidade de transplante } \\
\text { de medula óssea. }{ }^{17} \\
\text { Português e inglês } \\
2015\end{array}$ & \begin{tabular}{|l} 
Fermo VC, \\
Radünz V, \\
Rosa LM de, \\
Marinho MM/
\end{tabular} & $\begin{array}{l}\text { Avaliar a cultura de segurança } \\
\text { do paciente sob a ótica dos } \\
\text { profissionais da área de saúde } \\
\text { da unidade de Transplante de } \\
\text { Medula Óssea do Centro de } \\
\text { Pesquisas Oncológicas, hospital } \\
\text { de referência no tratamento do } \\
\text { câncer SC, Brasil }\end{array}$ & $\begin{array}{l}\text { Entre as dimensões analisadas, } \\
\text { somente a "satisfação no trabalho" } \\
\text { alcançou a média de escore acima } \\
\text { de } 75, \text { avaliada como positiva para a } \\
\text { cultura de segurança do paciente. }\end{array}$ \\
\hline 3 & $\begin{array}{l}\text { Fatores associados ao } \\
\text { Clima de segurança } \\
\text { do paciente em um } \\
\text { hospital de ensino. }{ }^{18} \\
\text { Português e inglês } \\
2015\end{array}$ & $\begin{array}{l}\text { Luiz RB, Simões } \\
\text { AL de A, } \\
\text { Barrichello E } \\
\text { Barbosa MH / }\end{array}$ & $\begin{array}{l}\text { Verificar a associação entre os } \\
\text { escores do clima de segurança } \\
\text { do paciente e as variáveis sócio- } \\
\text {-demográficas e profissionais. }\end{array}$ & $\begin{array}{l}\text { A maioria dos participantes era } \\
\text { do sexo feminino, da equipe de } \\
\text { enfermagem, atuavam na assistência } \\
\text { direta com pacientes adultos, em } \\
\text { áreas críticas, não possuíam pós- } \\
\text { graduação e nem apresentavam } \\
\text { outro vínculo empregatício. A } \\
\text { média e a mediana do escore geral } \\
\text { do instrumento foram de } 61,8 \\
\text { (DP=13,7) e } 63,3 \text {, respectivamente. } \\
\text { Encontrou-se como fator associado } \\
\text { ao clima de segurança a variável } \\
\text { atuação profissional para o domínio } \\
\text { percepção da gerência da unidade e } \\
\text { do hospital }(p=0,01)\end{array}$ \\
\hline 4 & $\begin{array}{l}\text { Cultura de Segurança } \\
\text { em um hospital de } \\
\text { ensino: diferenças de } \\
\text { percepção existentes } \\
\text { nos diferentes cenários } \\
\text { dessa instituição }{ }^{19} \\
\text { Português e inglês } \\
2015\end{array}$ & $\begin{array}{l}\text { Batalha EMSS, } \\
\text { Melleiro MM }\end{array}$ & \begin{tabular}{|l|} 
Avaliar a percepção dos \\
profissionais de enfermagem a \\
cerca da cultura de segurança do \\
paciente e identificar diferentes \\
de percepções nas unidades do \\
hospital
\end{tabular} & $\begin{array}{l}\text { Constatou-se que havia diferenças } \\
\text { significativas entre as unidades, } \\
\text { destacando-se as unidades } \\
\text { pediátricas que tiveram melhores } \\
\text { percepções de segurança (média: } \\
\text { 3,43 e mediana: } 3,44 \text { ). }\end{array}$ \\
\hline
\end{tabular}


Quadro 1. Continuação

\begin{tabular}{|c|c|c|c|c|}
\hline $\begin{array}{c}\text { Identificação } \\
\text { do Artigo }\end{array}$ & $\begin{array}{c}\begin{array}{c}\text { Título do artigo/ } \\
\text { Idioma/Ano }\end{array} \\
\end{array}$ & Autores & Objetivos & Desfecho \\
\hline 5 & $\begin{array}{l}\text { Clima de segurança do } \\
\text { paciente: percepção } \\
\text { dos profissionais de } \\
\text { enfermagem. }{ }^{20} \\
\text { Português e inglês } \\
2012\end{array}$ & $\begin{array}{l}\text { Rigobello MCG, } \\
\text { Carvalho REFL, } \\
\text { Cassiane SHde B, } \\
\text { Galon T, } \\
\text { Capucho HC, } \\
\text { Deus NN de/ }\end{array}$ & $\begin{array}{l}\text { Avaliar a percepção do clima } \\
\text { de segurança dos profissionais } \\
\text { de enfermagem atuantes nas } \\
\text { Clínicas médicas e cirúrgicas em } \\
\text { um hospital de ensino. }\end{array}$ & $\begin{array}{l}\text { A percepção do clima de segurança } \\
\text { dos profissionais variou conforme } \\
\text { o gênero, a clínica, a categoria } \\
\text { profissional e o tempo de } \\
\text { atuação. A satisfação no trabalho } \\
\text { foi demonstrada por todos os } \\
\text { profissionais, com escores acima de } \\
75 \text {, enquanto o domínio Percepção } \\
\text { da Gerência apresentou valores mais } \\
\text { baixos. }\end{array}$ \\
\hline 6 & $\begin{array}{l}\text { Comunicação e } \\
\text { segurança do } \\
\text { paciente: Percepção } \\
\text { dos profissionais de } \\
\text { enfermagem de um } \\
\text { hospital de ensino.. } \\
\text { Português e inglês } \\
2015\end{array}$ & $\begin{array}{l}\text { Massoco ECP, } \\
\text { Melleiro MM }\end{array}$ & \begin{tabular}{|l|} 
Conhecer a percepção de \\
trabalhadores de enfermagem \\
atuantes no hospital de \\
ensino acerca da dimensão da \\
abertura para as comunicações \\
e respostas não punitivas aos \\
erros e evidenciar a comunicação \\
como fator relevante na Cultura \\
de segurança do paciente
\end{tabular} & $\begin{array}{l}\text { Principais resultados do estudo, } \\
\text { identificou-se que os profissionais } \\
\text { conversam livremente sobre algo que } \\
\text { está errado. }\end{array}$ \\
\hline 7 & $\begin{array}{l}\text { Condutas adotadas } \\
\text { por Técnicos de } \\
\text { Enfermagem após a } \\
\text { ocorrência de Erros de } \\
\text { medicação.22 } \\
\text { Português e inglês }\end{array}$ & $\begin{array}{l}\text { Santos JO, } \\
\text { Silva AEBC, } \\
\text { Munari DB, } \\
\text { Miasso Al }\end{array}$ & $\begin{array}{l}\text { Identificar e analisar s condutas } \\
\text { por Tecs. de Enfermagem } \\
\text { após a ocorrência de erros de } \\
\text { medicação. }\end{array}$ & $\begin{array}{l}\text { Os resultados evidenciaram duas } \\
\text { categorias: tomando condutas } \\
\text { relacionadas à comunicação do erro } \\
\text { (ao médico, à enfermeira, registrando } \\
\text { no prontuário e não comunicando } \\
\text { o erro) e adotando condutas } \\
\text { direcionadas ao paciente (observação } \\
\text { do paciente, monitorização e } \\
\text { minimização das consequências) }\end{array}$ \\
\hline
\end{tabular}

Os autores do artigo $2^{17}$ utilizaram o SAQ para avaliar Cultura de segurança do paciente em uma unidade de TMO (Transplante de Medula Óssea) do Hospital do Câncer em Santa Catarina e apenas a dimensão "satisfação no trabalho" alcançou média de escore 75 avaliada como positiva para a Cultura de Segurança. Pelo estudo as demais dimensões presentes no inquérito necessitam ser valorizadas por profissionais e gestores para a efetivação de um cuidado seguro.

Já no artigo $3^{18}$, os pesquisadores verificaram a associação entre os escores do clima de segurança do paciente e as variáveis sócio demográficas, também utilizando o SAQ como instrumento. 0 estudo demonstrou que a identificação dos fatores associada ao clima de segurança facilita a elaboração de estratégias para práticas seguras nos hospitais.

Observa-se no estudo $4^{19}$ a aplicação do instrumento HSOPSC para avaliar a percepção dos profissionais acerca da Cultura de segurança e identificar as diferenças de percepção entre todas as unidades estudadas do hospital. Os autores constataram que havia diferenças significativas entre as unidades, e destas, as unidades pediátricas apresentaram melhores resultados para a percepção de segurança.

Por outro lado, a pesquisa $5^{20}$ avaliou a percepção do clima de segurança dos profissionais de enfermagem das clínicas médicas e cirúrgicas de um hospital de ensino, utilizando o SAQ. O estudo aponta que conhecer a percepção dos profissionais de enfermagem a respeito do Clima de segurança agrega melhorias ao cuidado em saúde e redução de riscos ao paciente.

Os pesquisadores do artigo $6^{21}$ estudaram a percepção da enfermagem acerca da dimensão da abertura para as comunicações e respostas não punitivas aos erros e a relevância da comunicação na Cultura de segurança do paciente. Para isso, foi utilizado o instrumento SHOPSC. 0 estudo demonstrou que os profissionais conversam sobre algo que está errado, porém, há receio de punição e da evidência do profissional, e esses são fatores limitantes na comunicação dos erros e da notificação dos eventos adversos.

Objetivando identificar e analisar condutas adotadas por Técnicos de enfermagem após a ocorrência de erros de medicação de um hospital de ensino em Goiânia$\mathrm{GO}$, os autores do artigo $7^{22}$ se utilizaram de entrevista semiestruturada e evidenciaram duas categorias. A primeira, refere-se às condutas em relação à comunicação do erro e a outra, às condutas direcionadas ao paciente. Percebeu-se que há necessidade das instituições de saúde adotarem uma cultura de transparência (padronização das notificações) em relação aos erros de medicação, direcionando as condutas dos profissionais e os auxiliando nas tomadas de decisões.

\section{2 - A pesquisa científica e sua contribuição na avaliação da Cultura de Segurança do paciente.}

Nessa segunda categoria destacam-se os artigos que retratam o cenário atual da Cultura de segurança do 
paciente nos hospitais de ensino pesquisados, abordando iniciativas, experiências exitosas, estratégias adotadas, dificuldades e barreiras encontradas.
O Quadro 2 apresenta os 6 artigos que a compõem, dispostos pelas mesmas variáveis do Quadro 1: Título, Periódico, Ano, Autores, Idioma e Objetivos.

Quadro 2. A pesquisa científica e sua contribuição na avaliação da Cultura de Segurança do paciente. Rio de Janeiro, RJ, Brasil, 2016.

\begin{tabular}{|c|c|c|c|c|}
\hline $\begin{array}{l}\text { Identificação } \\
\text { do Artigo }\end{array}$ & $\begin{array}{l}\text { Título do artigo/ } \\
\text { Idioma/Ano }\end{array}$ & Autores & Objetivos & Desfecho \\
\hline 8 & $\begin{array}{l}\text { Identificação } \\
\text { do paciente nas } \\
\text { organizações de } \\
\text { saúde: uma reflexão } \\
\text { emergente. } .^{23} \\
\text { Português e inglês. } \\
2013\end{array}$ & $\begin{array}{l}\text { Tase TH, } \\
\text { Lourenção DCA, } \\
\text { Bianchini SM, } \\
\text { Tronchin oMR }\end{array}$ & $\begin{array}{l}\text { Destacar os elementos } \\
\text { constituintes do processo de } \\
\text { identificação do paciente por } \\
\text { meio de pulseiras e refletir } \\
\text { acerca da implementação desse } \\
\text { processo nas instituições. }\end{array}$ & $\begin{array}{l}\text { A identificação do paciente por } \\
\text { meio de pulseira é uma prática } \\
\text { recomendada internacionalmente, } \\
\text { porém há lacunas no que tange à } \\
\text { instituição de protocolos, à execução } \\
\text { efetiva e à avaliação do processo } \\
\text { para subsidiar ações gerenciais e } \\
\text { assistências }\end{array}$ \\
\hline 9 & $\begin{array}{l}\text { Higienização das mãos } \\
\text { e a segurança do } \\
\text { paciente pediátrico. }{ }^{24} \\
\text { Português, inglês e } \\
\text { espanhol. } \\
2013 \\
\end{array}$ & $\begin{array}{l}\text { Silva FM, } \\
\text { Porto TP, } \\
\text { Rocha PK, } \\
\text { Lessmann JC, } \\
\text { Cabral PFA } \\
\text { Schneider KLK } \\
\end{array}$ & $\begin{array}{l}\text { Avaliar se a higienização } \\
\text { das mãos realizada antes do } \\
\text { preparo e da administração de } \\
\text { medicamentos e fluidoterapia } \\
\text { seguem as diretrizes da OMS }{ }^{25} \text { e } \\
\text { da ANVISA }{ }^{26}\end{array}$ & $\begin{array}{l}\text { Constatou-se a baixa adesão à prática } \\
\text { de higienização das mãos e estrutura } \\
\text { inadequada, segundo as diretrizes } \\
\text { estabelecidas pela OMS e ANVISA }\end{array}$ \\
\hline 10 & \begin{tabular}{|l|} 
Os profissionais da \\
saúde e a higienização \\
das mãos: uma questão \\
de segurança do \\
paciente pediátrico. $^{28}$ \\
Português e inglês $^{2014}$
\end{tabular} & \begin{tabular}{|l} 
Botene DZA, \\
Pedro ENR \\
\end{tabular} & $\begin{array}{l}\text { Analisar como a formação } \\
\text { acadêmica sobre a higienização } \\
\text { das mãos contribui para } \\
\text { a segurança do paciente } \\
\text { pediátrico. }\end{array}$ & $\begin{array}{l}\text { Os resultados permitiram elencar } \\
\text { duas categorias temáticas: “A HM e a } \\
\text { formação acadêmica do profissional } \\
\text { de saúde"; e "A HM e a vida } \\
\text { profissional". Neste manuscrito será } \\
\text { apresentada a primeira. Constatou-se } \\
\text { que a formação acadêmica contribui } \\
\text { de forma pouco efetiva para a criação } \\
\text { de uma cultura de segurança do } \\
\text { paciente. }\end{array}$ \\
\hline 11 & $\begin{array}{l}\text { Análise do registro e } \\
\text { conteúdo de checklists } \\
\text { para cirurgia segura }{ }^{29} \\
\text { Português e inglês. } \\
2015\end{array}$ & $\begin{array}{l}\text { Amaya MR, } \\
\text { Maziero ECS, } \\
\text { Grittem L, } \\
\text { Cruz EDA }\end{array}$ & $\begin{array}{l}\text { Analisar e relacionar o registro } \\
\text { de informações dos checklists } \\
\text { com os objetivos do Programa } \\
\text { cirurgias seguras salvam vidas }\end{array}$ & $\begin{array}{l}99,8 \% \text { dos itens do checklist foram } \\
\text { verificados e o teor dos registros } \\
\text { evidenciam não garantia, por meio da } \\
\text { checagem documental, de elementos } \\
\text { de segurança relativos ao local } \\
\text { cirúrgico certo (objetivo 1), perdas } \\
\text { sanguíneas (objetivo 4), reação } \\
\text { alérgica (objetivo 5), retenção de } \\
\text { instrumentais/compressas (objetivo } \\
\text { 7), identificação de espécimes } \\
\text { cirúrgicos (objetivo 8) e comunicação } \\
\text { (objetivo 9) }\end{array}$ \\
\hline 12 & $\begin{array}{l}\text { Estratégias educativas } \\
\text { para melhorar a adesão } \\
\text { à identificação do } \\
\text { paciente. }{ }^{.0} \\
\text { Português e inglês } \\
2015\end{array}$ & $\begin{array}{l}\text { Hemesath MP, } \\
\text { Santos HB, } \\
\text { Torelly EMS, } \\
\text { Barbosa AdaA, } \\
\text { Magalhães AMM }\end{array}$ & $\begin{array}{l}\text { Analisar o impacto de ações } \\
\text { educativas nos resultados } \\
\text { do indicador de adesão à } \\
\text { verificação da pulseira de } \\
\text { identificação do paciente, antes } \\
\text { da realização de cuidados de } \\
\text { maior risco. }\end{array}$ & $\begin{array}{l}\text { A análise e o acompanhamento } \\
\text { do indicador de adesão ao uso da } \\
\text { pulseira de identifi cação do paciente } \\
\text { demonstraram uma tendência de } \\
\text { aumento do percentual, atingindo, } \\
\text { ao longo do período estudado, de } \\
42,9 \% \text { a } 57,8 \% \text {, entre janeiro e abril } \\
\text { de } 2013 \text {, e de } 81,38 \% \text { a } 94,37 \% \text {, entre } \\
\text { setembro e dezembro de } 2014\end{array}$ \\
\hline 13 & $\begin{array}{l}\text { Uso de pulseiras } \\
\text { de identificação de } \\
\text { pacientes internados } \\
\text { em um hospital } \\
\text { universitário. }{ }^{31} \\
\text { Português e inglês }\end{array}$ & $\begin{array}{l}\text { Hoffmeister LV, } \\
\text { Moura GMSS de }\end{array}$ & $\begin{array}{l}\text { Avaliar o uso da pulseira de } \\
\text { identificação em pacientes } \\
\text { hospitalizados em unidades } \\
\text { de internação de um hospital } \\
\text { universitário. }\end{array}$ & $\begin{array}{l}\text { 83,9\% dos pacientes encontravam- } \\
\text { se com a pulseira corretamente } \\
\text { identificada, 11,9\% possuíam a } \\
\text { pulseira de identificação com erros } \\
\text { e 4,2\% dos pacientes estavam } \\
\text { sem a pulseira. As principais } \\
\text { inconformidades encontradas nas } \\
\text { pulseiras de identificação foram } \\
\text { nomes incompletos, números de } \\
\text { registros diferentes, ilegibilidade dos } \\
\text { dados e problemas na integridade. }\end{array}$ \\
\hline
\end{tabular}


No artigo $8^{23}$, os pesquisadores destacaram elementos constituintes do processo de identificação do paciente por meio de pulseiras e implementação dessas nas instituições hospitalares.

É uma prática recomendada internacionalmente, além de ser um dos protocolos de segurança do paciente ${ }^{27}$, porém existem lacunas na implantação do protocolo tanto por parte dos profissionais de saúde, gestores, entidades de classe e do próprio usuário que interferem na estruturação da Cultura de segurança do paciente.

Em contrapartida, temos o estudo $13^{31}$, que também avalia o uso da pulseira de identificação em unidades de internação, e que evidenciou o compromisso dos profissionais no processo de identificação dos pacientes, por meio da alta taxa de conformidades das pulseiras.

Os autores do artigo $12^{30}$ analisaram o impacto de ações educativas nos resultados do indicador de adesão à verificação da pulseira de identificação de pacientes, antes da realização de cuidados de maior risco. Os achados evidenciaram que as estratégias educativas alavancaram a adesão dos profissionais à verificação da identificação do paciente, com repercussão favorável na Cultura de segurança do paciente.

Os estudos $9^{24}$ e $10^{28}$ pesquisaram a questão da higienização das mãos na segurança do paciente pediátrico. 0 estudo $10^{28}$ constatou que a formação acadêmica do profissional é pouco efetiva para a construção de uma Cultura de lavagem das mãos e o estudo $9^{24}$ aponta para baixa adesão à prática de higienização das mãos associada à estrutura inadequada nas instituições, conforme preconizado nas diretrizes estabelecidas pela OMS (Organização Mundial de Saúde) ${ }^{25}$ e ANVISA (Agência Nacional de Vigilância Sanitária)26.

Temos no estudo $11^{29}$ uma abordagem que analisa e relaciona o registro de informações dos checklists com os objetivos do Programa cirurgias Seguras salvam vidas ${ }^{27}$. Evidenciou-se elevada frequência dos itens respondidos, porém, a não completude dos registros pela equipe cirúrgica, permitiu identificar potenciais riscos cirúrgicos provenientes de ações de segurança não confirmadas, necessitando de melhorias nas ações em busca da qualificação da assistência para a promoção da Cultura de segurança.

\section{CONCLUSÕES}

O objetivo desse estudo foi alcançado a partir do momento que permitiu identificar a produção científica da enfermagem dos hospitais de ensino brasileiros em relação à Cultura de segurança do paciente, evidenciando ainda um crescimento expressivo dessa nos últimos três anos, período que coincide com a criação do Programa Nacional de Segurança do Paciente.

Da análise e discussão dos resultados surgiram dois eixos temáticos: 1- Avaliação da Cultura de Segurança do paciente por meio da realização de pesquisas sobre a temática: os pesquisadores utilizaram preditores (o SAQ e o HSOPSC) sobre escores de segurança do paciente como importante ferramenta para diagnosticar, planejar, intervir e executar atividades para melhorar os domínios que precisam ser aprimorados, além de evidenciar fatores intrínsecos e extrínsecos dos profissionais que precisam de atenção. 2 - A pesquisa científica e sua contribuição na avaliação da Cultura de Segurança do paciente: Os pesquisadores procuraram divulgar através da pesquisa questões ligadas às iniciativas, experiências exitosas, estratégias adotadas, dificuldades e barreiras encontradas em relação à Cultura de segurança do paciente nos hospitais de ensino.

Como limitação do estudo, pode-se apontar a restrição dos cenários aos hospitais de ensino brasileiros, sugerindo que novas pesquisas que utilizem o método de Revisão Integrativa ou Sistemática sejam realizadas incluindo hospitais de ensino em outros países para, dessa forma, preencher as lacunas existentes neste estudo.

Além disso, novos estudos podem mensurar o clima de segurança, explicar a presença de microculturas nas instituições de saúde e propor novas estratégias de discussão sobre o tema, buscando cada vez mais um cuidado de saúde seguro e livre de danos.

A Cultura de Segurança tem a prevenção de erros como prioridade para todo: alta liderança, gestores, profissionais, pacientes e familiares. E para que isso ocorra, é necessário que as instituições de saúde criem barreiras que melhorem o processo de trabalho dos profissionais, por meio do emprego de sistemas que impossibilitem a ocorrência do mesmo, além de tornar mais difícil para os profissionais fazerem o errado e mais fácil fazerem o certo.

É necessário que as Instituições de Saúde adotem uma Cultura de Aprendizado (aprender com os erros) e não uma Cultura de Punição, a qual busca instituir culpados por falhas eminentemente resultantes de sistemas mal estruturados e mal desenhados.

\section{REFERÊNCAS}

1. Ministério da Saúde, Fundação Oswaldo Cruz, Agência Nacional de Vigilância Sanitária. Documento de referência para o programa nacional de segurança do paciente. Brasília: Ministério da Saúde; 2014 [acesso em 08 mar 2016]: 7. Disponível em: http://proqualis.net/sites/proqualis.net/files/ documento_referencia_programa_nacional_seguranca.pdf

2. Kohn LT, Corrignan JM, Donaldson MS, editors. To err is human: building a safer health system. Washington: National Academy Press; 2001 [acesso em 04 mai 2016]. Disponível em: https:// www.researchgate.net/publication/200656918_To_Err_is_ Human_Building_a_Safer_Health_System

3. Ministério da Saúde (BR), Agência de Vigilância Sanitária. Resolução da Diretoria Colegiada - RDC n 36, de 25 de julho de 2013. Institui ações para a segurança do paciente em serviços de saúde e dá outras providências. Diário Oficial 
da União 26 jul 2013 [acesso em 20 mai 2015]; Disponível em: http://bvsms.saude.gov.br/bvs/saudelegis/anvisa/2013/ rdc0036_25_07_2013.html

4. Mendes W, Martins M, Rozenfeld S, Travassos C. The assessment of adverse events in brazilian hospitals. Int J Qual Health Care [Internet]. 2009 [acesso em 03 nov 2015]; 21(4):279-84. Disponível em: http://intqhc.oxfordjournals. org/content/21/4/279.long

5. Travassos C, Porto S, Martins M, Mendes W.A magnitude financeira dos eventos adversos em hospitais no Brasil. Rev Port Saúde Pública [Internet]. 2010 [acesso em 15 mar 2016]; Vol. Temático(10):74-80. Disponível em: http://www.cdi.ensp.unl.pt/ docbweb/multimedia/rpsp2010-t\%20seg\%20doente/8a\%20 magnitude\%20financeira\%20dos\%20eventos\%20adversos\%20 em\%20hospitais\%20no\%20brasil.pdf

6. Kunh AM, Youngberg BJ. The need for risk management to evolve and assure e culture of safety.Qual Safe Health Care [Internet]. 2002 [acesso em 5jul 2015]; 11:158-62. Disponível em: http://qualitysafety.bmj.com/content/11/2/158.full. pdf+html

7. Pedreira MLG. Enfermagem e segurança do paciente. In: Pedreira MLG, Harada MJCS. Enfermagem dia-a-dia segurança do paciente. São Caetano do Sul, SP: Yendis; 2009. p. 23-4.

8. Silva MJP, Egry EY, Ângelo M, Barbosa MAM, Sousa RMC, Castilho V, Lopes NA, Batista AO. Produção do conhecimento em enfermagem: da idéia da pesquisa à publicação em periódico qualificado. Rev Esc Enferm USP [Internet]. 2009 [acesso em 20 jan 2016]; 43(spe2): 1347-51. Disponível em: http://www.scielo.br/pdf/reeusp/v43nspe2/a35v43s2.pdf

9. Pedreira, MLG. Errar é humano: estratégias para a busca da segurança do paciente. In: Harada MJCS, Pedreira MLG, Peterlini MAS, Pereira SR. 0 erro humano e a segurança do paciente. 7. ed. São Paulo: Atheneu; 2006. p. 7.

10. Carlucho R, Nepomuceno L, Garofolo MM, Ferro MG, Almeida EF. Os HU's que temos e os hospitais universitários que queremos: regimento geral dos HU's, hospitais escolas e centros de saúde escola. Revista FASUBRA [Internet]. 2012 [citado em 06 abr 2016]; 7:1-17. Disponível em: http://www. ufam.edu.br/attachments/article/2317/Projeto_HUs.pdf

11. Cooper HM. The integrative research review: a systematic approach. Beverly Hills: Sage; 1984.

12. Mendes KDS, Silveira RCCP, Galvão CM. Revisão integrativa: método de pesquisa para a incorporação de evidências na saúde e na enfermagem. Texto \& contexto enferm [Internet]. 2008[acesso em 30 dez 2015]; 17(4):758-64. Disponível em: http://www.scielo.br/pdf/tce/v17n4/18.pdf

13. Souza MT, Silva MD, Carvalho R. Revisão integrativa: o que é e como fazer? Einstein [Internet] 2010 [acesso em 13 mar 2016]; 8(1 Pt 1):102-6. Disponível: http://www. astresmetodologias. com/material/O_que_e_RIL.pdf

14. Santiago THR, Turrini RNT. Cultura e clima organizacional para segurança do paciente em unidades de terapia intensiva. Rev. Esc Enferm USP [Internet]. 2015 [acesso em 12 mar 2016]; 49(spe):123-30. Disponível em: http://www.scielo.br/ pdf/reeusp/v49nspe/1980-220X-reeusp-49-spe-0123.pdf

15. Sorra J, Famolaro T, Dyer N, Nelson D, Khanna K; Agency for Healthcare Research and Quality. Hospital Survey on patient safety culture 2008: comparative database report. AHRQ n. 08-0039 [Internet].2008 [acesso em 21 jan 2016]. Disponível em: https://archive.ahrq.gov/professionals/quality-patientsafety/patientsafetyculture/hospital/2008/2008-hospitalsurvey.pdf

16. Sexton JB, Helmreich R, Neilands TB, Rowan K, Vella K, Boyden J, Roberts PR, Thomas E. The safety attitudes questionnaire: psychometric properties, benchmarking data, and emerging research. BMC Health Serv Res [Internet]. 2006 [acesso em 7jun 2015]; 44(6):1-10. Disponível em: https://www.ncbi.nlm. nih.gov/pmc/articles/PMC1481614/pdf/1472-6963-6-44.pdf

17. Fermo VC, Radünz V, Rosa LM, Marinho MM. Cultura de segurança do paciente em unidade de transplante de medula óssea. Rev bras enferm [Internet]. 2015 [acesso em $15 \mathrm{fev}$ 2016]; 68(6):1139-46. Disponível em: http://www.scielo.br/ pdf/reben/v68n6/0034-7167-reben-68-06-1139.pdf

18. Luiz RB, Simões ALA, Barichello E, Barbosa MHH. Fatores associados ao clima de segurança do paciente em um hospital de ensino. Rev latino am enferm [Internet]. 2015 set-out [citado em 08 mar 2016];23(5):880-7. Disponível em: $\quad$ http://www.scielo.br/pdf/rlae/v23n5/pt_0104-1169rlae-23-05-00880.pdf

19. Silva-Batalha EMS, Melleiro MM. Cultura de segurança do paciente em um hospital de ensino: diferenças de percepção existentes nos diferentes cenários dessa instituição. Texto \& contexto enferm [Internet]. 2015 [acesso em 08 mar 2016]; 24(2):432-41. Disponível em: http://www.scielo.br/pdf/tce/ v24n2/pt_0104-0707-tce-24-02-00432.pdf

20. Rigobello MCG, Carvalho REFL, Cassiani SHB, Galon T, Capucho HC, Deus NN. Clima de segurança do paciente: percepção dos profissionais de enfermagem. Acta paul enferm [Internet]. 2012 [acesso em 08 mar 2016]; 25(5):728-35. Disponível em: http://www.scielo.br/pdf/ape/v25n5/13.pdf

21. Massoco ECP, Melleiro MM. Comunicação e segurança do paciente: percepção dos profissionais de enfermagem de um hospital de ensino. REME rev min enferm [Internet]. 2015[citado em 10 mar 2016]; 19(2):187-91. Disponível em http://www.reme.org.br/artigo/detalhes/1014

22. Santos JO, Silva AEBC, Munari DB, Miasso Al. Condutas adotadas por técnicos de enfermagem após ocorrência de erros de medicação. Acta paul enferm[Internet]. 2010 [acesso em 08 abr 2016]; 23(3):328-33. Disponível em: http://www.scielo.br/scielo.php?script=sci_ arttext \& pid=S0103-21002010000300003

23. Tase TH,LourençãoDCA,BianchiniSM,Tronchin DMR.Identificação do paciente nas organizações de saúde: uma reflexão emergente. Rev. Gaúch enferm [Internet]. 2013[acesso em 10 mar 2016]; 34(3):196-200. Disponível em: http://www.scielo.br/scielo. php?script=sci_arttext\&pid=S1983-14472013000300025

24. Silva FM, Porto TP, Rocha PK, Lessmann JC, Cabral PFA, Schneider KLK. Higienização das mãos e a segurança do paciente pediátrico. Cienc enferm [Internet]. 2013 [acesso em 12 abr 2016]; (2):99-109. Disponível em http://www.scielo.cl/ pdf/cienf/v19n2/art_10.pdf

25. Organização Mundial da Saúde. Diretrizes da OMS sobre higienização das mãos na assistência à saúde (versão preliminar avançada): resumo. Geneva: WHO; 2005. 34 p.

26. Agência Nacional de Vigilância Sanitária (BR). Higienização das mãos em serviços de saúde. Brasília: Anvisa; 2007. 52 p.

27. Ministério da saúde (BR).Portarian ${ }^{\circ}$ 1.377, de 9 de julho de 2013. Aprova os Protocolos de Segurança do Paciente. Diário 
Oficial da União 10 jul. 2013. [acesso em 20 mai 2015]. Disponível em: http://bvsms.saude.gov.br/bvs/saudelegis/ gm/2013/prt1377_09_07_2013.html

28. Botene DZA, Pedro ENR. Os profissionais da saúde e a higienização das mãos: uma questão de segurança do paciente pediátrico. Rev. Gaúch enferm [Internet]. 2014 [acesso em 08 fev 2016]; 35(3):124-9. Disponível em: http://www.scielo.br/ pdf/rgenf/v35n3/pt_1983-1447-rgenf-35-03-00124.pdf

29. Amaya MR, Maziero ECS, Grittem L, Cruz EDA. Análise do registro e conteúdo de checklists para cirurgia segura. Esc Anna Nery Rev. Enferm [Internet]. 2015 [acesso em 8 mar 2016]; 19(2):246-51. Disponível em: http://www.scielo.br/ pdf/ean/v19n2/1414-8145-ean-19-02-0246.pdf

30. Hemesath MP, Santos HB, Torelly EMS, Barbosa AS, Magalhães AMM. Estratégias educativas para melhorar a adesão à identificação do paciente. Rev. Gaúch enferm [Internet]. 2015 [acesso em 09 Mar 2016]; 36(4):43-48. Disponível em: http://www.scielo.br/pdf/rgenf/v36n4/pt_1983-1447rgenf-36-04-00043.pdf

31. Hoffmeister LV, Moura GMSS. Uso de pulseiras de identificação em pacientes internados em um hospital universitário. Rev. Latino am enferm [Internet]. 2015 [acesso em 08 mar 2016]; 23(1): 3643. Disponível em: http://www.scielo.br/pdf/rlae/ v23n1/pt_0104-1169-rlae-23-01-00036.pdf 